\title{
Heiliger Stuhl, Drittes Reich und Radio Vaticana
}

\author{
Die Beziehungen zwischen dem Vatikan und dem \\ nationalsozialistischen Deutschland im Spiegel des päpstlichen \\ Rundfunksenders (1936-1943)
}

\begin{abstract}
This article intends to show that the German language broadcasts of Vatican Radio influenced relations between the Holy See and the Third Reich in the period between the start of the station's radio broadcasts and 1943. As emerges from the analysis of published and unpublished sources, Vatican Radio seems to have been used as a Catholic propaganda tool in Germany, but also as an instrument of diplomacy. Vatican radio continued to broadcast in German and to attract listeners even after Goebbels' decree of 1 September 1939 forbidding people to listen to foreign radio stations. The Holy See, aware that its broadcasts were being monitored by the Sonderdienst Seehaus and of their importance not only for the population of Germany but also for its government, exploited them to spread specific ideas and messages depending on the circumstances and the progress of the war. The study of Vatican Radio thus represents a specific point of view from which to understand the Holy See's attitude towards National Socialism and its actions during the Second World War.
\end{abstract}

\section{Eine methodologische Vorbemerkung}

Papst Franziskus’ Ankündigung, die lang erwartete Öffnung der vatikanischen Archive für die Zeit des Pontifikats von Pius XII. (1939-1958) stehe kurz bevor, ist vom Fachpublikum höchst positiv aufgenommen worden. ${ }^{1}$ Nicht alle Stimmen, die diese Nachricht in der Presse oder im Netz kommentiert haben, waren davon überzeugt, das neue Archivmaterial werde zu einer historischen Neubestimmung der Rolle des Heiligen Stuhls und der Orientierung von Papst Pacelli während des Zweiten Weltkrieges

\footnotetext{
1 Papstaudienz für die Archivbelegschaft am 4.3.2019 (http://press.vatican.va/content/salastampa/ it/bollettino/pubblico/2019/03/04/0185/00375.html; 20.9.2020). Der vorliegende Beitrag wurde der Redaktion vor der für März 2020 vorgesehenen Öffnung der Bestände des vatikanischen Archivs zum Pontifikat Pius’ XII zur Drucklegung übergeben.
}

Hinweis: Übersetzung von Gerhard Kuck.

Kontakt: Raffaella Perin, raffaella.perin@unicatt.it 
führen. Die einen meinten, nun würden „endlich die Motive, die seinen Entscheidungen zugrunde lagen“, geklärt, ${ }^{2}$ die anderen bezweifelten, dass sich das solide Bild, wie es der Triester Historiker Giovanni Miccoli in „I dilemmi e i silenzi di Pio XII“ auf der Grundlage der von der Kommission für Zeitgeschichte edierten deutschen Quellen ${ }^{3}$ und der „Actes et documents du Saint-Siège relatifs à la seconde guerre mondiale“4 gezeichnet hatte, wesentlich ändern werde. ${ }^{5}$ Ich neige der zweiten Ansicht zu, auch wenn das neue Archivmaterial zweifellos das Wissen über dieses Pontifikat bereichern und damit erlauben wird, einige Fragestellungen schärfer zu fassen und das Gesamturteil über die vom Heiligen Stuhl während des Krieges vertretene Linie auszudifferenzieren.

Der vorliegende Beitrag befasst sich mit den deutschsprachigen Sendungen und dem Einfluss, den sie auf das Verhältnis zwischen dem Heiligen Stuhl und dem Dritten Reich von 1936 bis 1943 ausübten, und vertieft damit einen Aspekt meiner umfänglicheren Studien zum Vatikansender von seinen Anfängen bis zum Zweiten Weltkrieg. ${ }^{6}$ Angesichts der Tatsache, dass die noch gesperrten Archivbestände nicht zugänglich waren, ist eine methodologische Vorbemerkung zum Forschungsstand und zu den Schwierigkeiten bei der Quellenbeschaffung unerlässlich.

Die Geschichte der Rundfunkanstalt wurde bisher zumeist auf populärwissenschaftlicher Ebene oder in apologetischer Absicht behandelt. Jeder neue historische Mosaikstein, der ihre Rolle während des Krieges beleuchtet, wird dementsprechend die große strategische Bedeutung unterstreichen, die der Heilige Stuhl ihr in propagandistischer und diplomatischer Hinsicht beimaß, und dazu beitragen, Figur und Wirken Papst Pius' XII. neu zu überdenken und die Kenntnisse über ihn zu vertiefen.

Die Komplexität des hierzu notwendigen Archivmaterials stellte eine große Herausforderung dar und verlangte die Verknüpfung verschiedener partieller, von

2 Interview mit Andrea Riccardi, La Stampa, 5.3 .2019 (https://www.lastampa.it/2019/03/05/vatican insider/riccardi-non-c-nulla-da-temere-dallapertura-degli-archivi-di-pio-xii-lRvdDh4xDKhf iXBsWGVE9M/pagina.html; 20.9.2020).

3 Seit den 60er Jahren förderte die Kommission für Zeitgeschichte die Veröffentlichung von Dokumenten zur Lage der deutschen Kirche unter dem nationalsozialistischen Regime.

4 Actes et documents du Saint-Siège relatifs à la seconde guerre mondiale (= ADSS), 11 Bde., hg. von Pierre Blet u. a., Città del Vaticano 1965-1981.

5 Daniele Menozzi, Archivi vaticani: la Chiesa non teme la storia, in: Settimana News (http://www. settimananews.it/chiesa/archivi-vaticani-la-chiesa-non-teme-la-storia/; 20.9.2020). Gemeint ist die Studie von Giovanni Miccoli, I dilemmi e i silenzi di Pio XII. Vaticano, Seconda guerra mondiale e Shoah, Milano 22007.

6 Aus jenen Studien sind einige Artikel, darunter Raffaella Perin, Vatican Radio and Modern Propaganda. A Case Study, in: The Papacy in the Contemporary Age, hg. von Giovanni Vian, Venezia 2018, S. 67-86 (https://edizionicafoscari.unive.it/libri/978-88-6969-256-7/vatican-radio-and-modernpropaganda/; 20.9.2020), und die Monographie Raffaella Perin, La radio del papa. Propaganda e diplomazia nella seconda guerra mondiale, Bologna 2017, erwachsen. Ein Stipendium des Deutschen Historischen Instituts in Rom vom 1. September bis zum 31. Dezember 2016 hat die vertiefenden Nachforschungen, Grundlage dieses Beitrags, ermöglicht. Mein Dank geht an den Direktor des Instituts Martin Baumeister und an seinen Stellvertreter Lutz Klinkhammer. 
unterschiedlichen Organen produzierter Quellen. Tatsächlich befinden sich die Sendemanuskripte nicht, wie man vermuten könnte, im Archiv des Senders. Ihre Rekonstruktion beruht auf einer Kombination von Mitschriften, die von den Überwachungsdiensten der ausländischen Rundfunkanstalten, insbesondere der BBC in England und des Sonderdienstes Seehaus in Deutschland, vorgenommen wurden. Die resümierten Inhalte stammen also aus der Feder der Empfänger und Hörer, nicht der Produzenten. Ihre Verlässlichkeit ergibt sich daraus, dass sie für den internen Gebrauch bestimmt waren und an die verschiedenen Ministerien zur weiteren Verwendung gingen. Es gibt keinen vernünftigen Grund anzunehmen, dass sie wissentlich verfälscht wurden, auch wenn sich mögliche Verständnisfehler seitens der Abhördienste nicht ganz ausschließen lassen. Soweit es hingegen die Beziehungen zwischen dem Heiligen Stuhl und dem Dritten Reich mit Blick auf Radio Vaticana betrifft, habe ich neben den bereits erwähnten Sammlungen der Kommission für Zeitgeschichte und der „Actes et documents“ zumeist auf das traditionelle Quellenmaterial der Kirchenarchive und religiösen Einrichtungen zurückgegriffen (u. a. Archivio Apostolico Vaticano, Affari Ecclesiastici Straordinari, Archiv des Jesuitenordens).

Die Fragen, deren Beantwortung hier versucht wird, richten sich auf die folgenden Aspekte: Welche Rolle spielte der Vatikansender während des Krieges im Rahmen der propagandistischen und diplomatischen Strategie des Heiligen Stuhles? Auf welche Weise beeinflusste er die Beziehungen, die der Heilige Stuhl zur deutschen Diplomatie unterhielt? Bedingte er, und wenn ja, in welchem Maße, die Religionspolitik des Hitlerregimes? Lässt sich bestimmen, bis zu welchem Umfang die Übertragungen von Radio Vaticana im Reich und in den von Deutschland besetzten Territorien empfangen wurden?

\section{Die frühen Jahre (1936-1939)}

Mit der Eröffnung von Radio Vaticana am 12. Februar 1931 durch Pius XI. konnte man zum ersten Mal die Stimme des Papstes in der ganzen Welt vernehmen. Als Sitz wählte man die vatikanischen Gärten in der Nähe der Specola Vaticana, die Leitung wurde den Jesuiten anvertraut, die auch die gesamte Redaktion stellten. Die eigentliche Rundfunktätigkeit begann jedoch erst 1936 und wurde von diesem Zeitpunkt an immer regelmäßiger betrieben. Gesendet wurde im zweiwöchigen Wechsel auf Italienisch, Deutsch, Französisch, Spanisch und Englisch (mit unterschiedlichen Inhalten für die Vereinigten Staaten, den englischsprachigen Orient und Großbritannien), später auch auf Holländisch, Polnisch und Russisch. ${ }^{7}$

7 Perin, La radio del papa (wie Anm. 6), S. 19-48; Fernando Bea/Alessandro De Carolis, Ottant'anni della radio del papa, Bd. 1, Città del Vaticano 2011; Alberto Monticone, La radio vaticana tra fascismo e guerra (1931-1944), in: Chiesa e società dal secolo IV ai nostri giorni. Studi storici in onore del P. Ilarino da Milano, Bd. 2, Roma 1979, S. 681-727. 
Die Frage, wer für die Inhalte verantwortlich zeichnete, ist von höchster Bedeutung und spielte eine entscheidende Rolle für die Beziehungen zum Reich. Soweit sich erkennen lässt, war die Redaktion zwischen 1936 und 1939 im wesentlichen unabhängig vom Heiligen Stuhl, brauchte also weder dem Staatssekretariat noch dem Papst Rechenschaft über das von ihr gestaltete Programm abzulegen; auf keinen Fall wurden diese regelmäßig informiert. Nach Kriegsausbruch begann Pius XII., der im Frühjahr 1939 zum Papst gewählt worden war, das neue Medium bewusster einzusetzen, sah er doch, dass es für seine von den kriegführenden Ländern aufmerksam verfolgte Politik von Nutzen sein konnte. Anfang Oktober 1940 änderten sich dann die allgemeinen Rahmenbedingungen. Aufgrund der anhaltenden Klagen seitens der deutschen Botschaft beim Heiligen Stuhl über die Inhalte wurden die Sendemanuskripte zunächst nach der Übertragung zur Kenntnisnahme an das Staatssekretariat übermittelt. Schließlich bewirkten die Drohungen und Repressalien von deutscher Seite, dass im Juni 1941 eine Präventivzensur eingeführt und zu diesem Zweck im Staatssekretariat die Stelle eines Zensors geschaffen wurde. ${ }^{8}$

Die deutschsprachigen Sendungen besaßen von Anfang an eine besondere Bedeutung. Radio Vaticana konnte in der Tat als Propagandainstrument für die deutschen Länder und deutschsprachigen Gebiete dienen, nachdem das Verbot der katholischen Presse und die Entfernung der Katholiken aus den deutschen Rundfunkanstalten die Verbreitung von praktisch allen Informationen aus der Kirchenwelt unterbunden hatte. ${ }^{9}$ In einem vom Chef des Sicherheitsamtes im Mai - Juni 1934 verfassten Bericht heisst es, die deutschen Katholiken hörten die ausländischen Sender, weil sie Nachrichten erhalten wollten, die über rein religiöse Themen hinausgingen, auf die sich die katholischen Medien in Deutschland zu beschränken hatten; auch würden sie Kurzwellenempfänger vor allem deshalb kaufen, um Radio Vaticana hören zu können. Ebenso scheint hier die Befürchtung der nationalsozialistischen Regierung durch, dass die religiösen Rundfunkanstalten unter dem Vorwand, religionsspezifische Gegenstände zu behandeln, Propaganda für den sogenannten politischen Katholizismus betrieben. ${ }^{10}$

Der Münchner Erzbischof Kardinal Michael von Faulhaber ${ }^{11}$ hatte wiederholt anklingen lassen, dass er die vatikanische Sendeanstalt für wichtig halte. Während einer Audienz legte er Papst Pius XI. nahe, auch positive Nachrichten aus Deutschland

8 Perin, La radio del papa (wie Anm. 6), S. 101-118.

9 Vgl. Gunther Lewy, I nazisti e la Chiesa, Milano 22013, S. 195; Alessandro Bellino, Il Vaticano e Hitler. Santa Sede, Chiesa tedesca e nazismo (1922-1939), Milano 2019, S. 172.

10 Berichte des SD und der Gestapo über Kirchen und Kirchenvolk in Deutschland 1934-1944, bearb. von Heinz Boberach, Mainz 1971, Dok. 1: Lagebericht des Chefs des Sicherheitsamtes des Reichsführers SS, S. 3-63, hier S. 38: „Katholische Kreise kaufen vorwiegend Empfangsapparate mit Kurzwellenempfangsteilen, vor allem wohl, um den Vatikansender abhören zu können.“.

11 Die Edition der Tagebücher Faulhabers ist in Arbeit (https://www.faulhaber-edition.de/index. html; 20.9.2020). 
zu bringen; er selbst wolle veranlassen, dass entsprechendes Material zur Verfügung gestellt werde. ${ }^{12}$ In einem Gespräch zwischen Pacellis Privatsekretär Pater Robert Leiber, dem Freiburger Erzbischof Conrad Gröber und dem Exponenten der Zentrumspartei Albert Hackelsberger, das im August 1937 in Riedenburg stattfand, wurde die Notwendigkeit hervorgehoben, Radio Vaticana solle nicht nur religiöse Fragen behandeln, sondern sich auch zu aktuellen Problemen äußern. Zum Beispiel könne man Artikel aus dem „Schwarzen Korps“ oder Pressemitteilungen bestimmter nationalsozialistischer Minister wiedergeben, die keines weiteren Kommentars bedürften und die verfehlte Politik gegenüber der Kirche in Deutschland deutlich machten. Außerdem lasse sich das Potential des Radiosenders nutzen, um Falschnachrichten zu widerlegen oder - gern gehörte - Musik zu übertragen. ${ }^{13}$

Zum ersten großen Konflikt zwischen der deutschen Regierung und dem Heiligen Stuhl bezüglich des Radiosenders kam es im Zusammenhang mit dem Anschluss Österreichs. ${ }^{14}$ Nach Veröffentlichung der „Feierlichen Erklärung“ des österreichischen Episkopats vom 18. März 1938, worin die Errungenschaften des Nationalsozialismus gepriesen und die österreichischen Katholiken aufgefordert wurden, am 10. April bei der Volksabstimmung für den Anschluss zu stimmen, hatte sich der Heilige Stuhl in einer Note, die am 1. April im „Osservatore Romano“ erschien, gegen die Position der österreichischen Bischöfe ausgesprochen. ${ }^{15}$ Auch die vatikanische Rundfunkanstalt setzte sich in einer deutschsprachigen Sendung kritisch mit ihr auseinander. Der Sprecher griff am selben Tag den „falschen politischen Katholizismus“ an, worunter

12 Akten Kardinal Michael von Faulhabers 1917-1945, Bd. 2: 1935-1945, bearb. von Ludwig Volk, Mainz 1978, Dok. 609, S. 282-284, hier S. 283. Der Kardinal griff das Thema noch einmal in einem Brief an den Priester Emil Muhler aus der Diözese München auf: Er habe seine Auffassung, wonach Presse und Rundfunkanstalt zu negativ arbeiteten und es nicht schafften, die katholische Lehre in ein positives Licht zu rücken, „auch dem Vatikanischen Sender gegenüber zum Ausdruck gebracht“; ebd., Dok. 613, S. 296f. Zu Muhler vgl. Deutsche Biographie (https://www.deutsche-biographie.de/ gnd129414360.html\#ndbcontent; 20.9.2020).

13 Akten deutscher Bischöfe über die Lage der Kirche 1933-1945, Bd. 4: 1936-1939, bearb. von Ludwig Volk, Mainz 1981, Dok. 398, S. 267-272.

14 Vgl. Georges Passelecq/Bernard Suchecky, L'encyclique cachée de Pie XI. Une occasion manquée de l'Église face à l'antisémitisme, Paris 1995, S. 96-103; Paolo Valvo, Dio salvi l'Austria! 1938: il Vaticano e l'Anschluss, Milano 2010, S. 181 f.; ders., Hitler, il nunzio e il cardinale. Il memoriale di mons. Gaetano Cicognani del 12 aprile 1938, in: Nuova storia contemporanea 6 (2012), S. 69-87.

15 Die Notiz wurde am 1. April 1938 während einer Audienz des damaligen Staatssekretärs Pacelli bei Pius XI. verfasst. „Aufgrund der verschiedenen, oftmals tendenziösen Kommentare (auch von unerwarteter Seite) zur bekannten Erklärung des österreichischen Episkopats werden wir beauftragt, als Tatsache und unabhängig von jeglicher politischer Erwägung und Problemstellung mitzuteilen, dass sie unter alleiniger Verantwortung desselben Episkopats entworfen und unterzeichnet wurde, ohne sich vorher mit dem Heiligen Stuhl zu verständigen oder dessen nachträgliche Genehmigung einzuholen." Segreteria di Stato, Sezione per i Rapporti con gli Stati, Archivio Storico, Archivio della Sacra Congregazione per gli Affari Ecclesiastici Straordinari (SRSS AAEESS), Stati Ecclesiastici, 430a P. O., fasc. 355. 
er die sowohl von den einfachen Gläubigen als auch den Vertretern der Amtskirche im öffentlichen Leben eingenommene Haltung verstand:

\begin{abstract}
„Dieser falsche politische Katholizismus ist vielmehr eine Art und Weise des Verhaltens der Katholiken, sei es des einfachen Gläubigen, sei es des Amtsträgers im öffentlichen Leben, die lediglich aus überkluger Vorsicht und Taktik und schwächlicher Anpassung an gegebene oder erwartete Tatsachen besteht. ... Am grössten war und ist der Schaden dann, wenn sogar die berufenen Hirten der göttlichen Sittenordnung von jenem Geist des falschen politischen Katholizismus erfasst waren oder sind, und zwar irgendwie befangen unter dem Eindruck der Mächtigen und Erfolgreichen des Tages. Dann mag es kommen, dass die Augen solcher Hirten nicht mehr, wie es doch eigentlich ihre Pflicht wäre, den Wolf im Schafspelz erkennen, und dass sie Versprechungen von Menschen glauben, vor denen sie die traurigen Erfahrungen anderer, ja sogar das Wort des obersten Hirten selbst hätte warnen müssen. Die Folge dieser Haltung wird dann stets sein, dass solche Hüter der kirchlichen Interessen sich wirkliche und beklagenswerte Übergriffe vom religiös-sittlichen auf das ausschliessliche politische Gebiet zuschulden kommen lassen. Sie nützen beispielsweise ihre religiöse Stellung aus, um die Gläubigen von der Wahrheit bestimmter Behauptungen in rein praktischen Dingen des Politisch-Gesellschaftlichen zu überzeugen, selbst dann, wenn jene Behauptungen und die ihnen zugrundliegenden Tatsachen von vielen besonnenen und sachverständigen Menschen anders beurteilt werden. Beispielsweise ist es nicht Sache der kirchlichen Lehrautorität als solcher, Erklärungen abzugeben, welche die rein wirtschaftlichen, sozialen und volkspolitischen Erfolge einer Regierung messen und werten. Kein Gläubiger ist im Gewissen verpflichtet, diesem Urteil als Urteil der kirchlichen Lehrautorität seine Zustimmung zu geben und den Gebrauch seiner politischen Rechte danach zu orientieren." ${ }^{\prime 16}$
\end{abstract}

Verfasser des Sendemanuskripts war Pater Gustav Gundlach (der auch an der sogenannten geheimen Enzyklika über Rassismus und Antisemitismus mitwirkte), er hat es aber aller Wahrscheinlichkeit nach nicht selbst am Mikrofon verlesen. ${ }^{17}$ Nach Ansicht des „Catholic Herald“ trug ein gewisser Pater Immer den Text vor. Der „Osservatore Romano“ bemerkte am selben Tag: „Die Nachforschungen und Nachrichten [über die Sendung] gingen in eine andere Richtung, sie legten nahe, dass der Jesuitenpater, der für gewöhnlich die deutschsprachigen Nachrichten am Mikrofon verliest, Autor des Beitrags sei, so als ob die Radiosprecher in allen Ländern die Autoren dessen seien, was sie dort verlesen.“ Der „Catholic Herald“ fügte hinzu: „Though this broadcast was disclaimed by the Vatican it is pointed out that this does not imply disagreement with the statement as a private opinion of relevance to the situation. In future Vatican broadcasts will be more strictly censored.“

Beschwerden seitens des deutschen und italienischen Botschafters ließen nicht lange auf sich warten. Unter dem 2. April notierte der Sekretär der Kongregation für die außerordentlichen kirchlichen Angelegenheiten, Monsignor Domenico Tardini, der italienische Botschafter Pignatti habe ihn angerufen und angeraten, durch den „Osservatore Romano“ die Meldung dementieren zu lassen, wonach das Staatssekre-

16 SRSS AAEESS, Austria, pos. 912-914 P. O., fasc. 71, fol. 10-13.

17 Vgl. Vatican and Austrian Bishops’ Declaration: in: The Catholic Herald, 8.4.1938, S. 1. 
tariat die Sendung genehmigt hatte; andernfalls würden alle davon ausgehen, dass es dafür verantwortlich zeichne, ja dem Papst selbst die Urheberschaft zuschreiben. ${ }^{18}$ Am Nachmittag desselben Tages war der Botschaftsrat der italienischen Botschaft beim Heiligen Stuhl im Staatssekretariat vorstellig geworden und hatte um Erklärungen gebeten; die Antwort lautete, die Radioübertragung habe weder einen „offiziellen noch offiziösen Charakter“ und das Staatssekretariat „keinerlei Kenntnis vom Text“ gehabt. Am Abend wurde noch einmal bekräftigt, dass es sich um ,eine rein private Initiative“ gehandelt und „das Staatssekretariat mit dem, was übertragen wurde, nichts zu tun“ gehabt habe. ${ }^{19}$ In seinen Memoiren hingegen schreibt Gundlach: „Der Kardinalstaatssekretär Pacelli, der die Sendung überprüft und gebilligt hatte, wurde von Pius XI. voll gedeckt.“20

Die Reichsbehörden beobachteten die Sendetätigkeit von Radio Vaticana von Anfang an mit höchster Wachsamkeit, wobei sich die Polizei auf ein Netz von Informanten stützen konnte. So teilte der bayerische Regierungspräsident Hans Dippold in seinem Bericht zum Dezember 1938 mit, im Kreis Hilpoltstein seien in letzter Zeit verschiedene teure Kurzwellenempfänger erworben worden, um dienstags und freitags von 20.30 bis 21.00 Uhr die Sendungen der vatikanischen Rundfunkanstalt verfolgen zu können. Nicht nur einige Diözesanblätter, auch die Pfarrer hätten von der Kanzel herab geraten, sich die Übertragungen anzuhören. ${ }^{21}$ Während es in einem anderen Bericht des bayerischen Regierungspräsidenten heißt, die vatikanische Rundfunkanstalt habe sondiert, ob die Sendungen gut empfangen werden. ${ }^{22}$ Die Ergebnisse dieser Umfrage lassen sich in einem Bericht des Direktors P. Filippo Soccorsi an Pacelli aus dem Jahr 1939 nachlesen, wonach ungefähr viertausend Briefe aus Deutschland eingegangen waren. ${ }^{23}$ Berücksichtigt man die Gesamtzahl der deutschen Katholiken, hatte einer von fünftausend potentiellen Hörern geantwortet; und da sich gewiss nicht alle die Mühe gemacht haben werden, zur Feder zu greifen, kann man von einer höheren Hörerzahl ausgehen. Pater Soccorsi notierte in seinem Bericht:

18 SRSS AAEESS, Austria, pos. 912-914 P. O., fasc. 71, fol. $18 \mathrm{f}$.

19 Ibid., fol. $24 \mathrm{f}$.

20 Zitiert nach Johannes Schwarte, Gustav Gundlach S. J. (1892-1963). Maßgeblicher Repräsentant der katholischen Soziallehre während der Pontifikate Pius XI. und Pius XII., München-PaderbornWien 1975, S. 69.

21 Die Kirchliche Lage in Bayern nach den Regierungspräsidentenberichten 1933-1943, Bd. 2, bearb. von Helmut Witetschek, Mainz 1967, Dok. 92: Monatsbericht der Regierung (Dezember 1938), Ansbach, 7. Januar 1939, S. 304 f.: „Im Bezirk Hilpoltstein konnte die Wahrnehmung gemacht werden, daß in letzter Zeit verschiedentlich teuere Kurzwellengeräte (Marke Lumophon WD 469) gekauft wurden, weil mit diesen auf Welle 49 an den Dienstagen und Freitagen von 20.30 bis 21.00 Uhr der Vatikansender gehört werden kann. Es ist bezeichnend, daß solche Apparate hauptsächlich Personen beschafft haben, die in enger Beziehung zum Pfarrhof stehen. Daß von dort die Radioempfehlung ausgeht, ist wohl mit Sicherheit anzunehmen.“.

22 Ebd., Dok. 95, S. 313.

23 Promemoria 25 marzo 1939, in: Archivum Romanum Societatis Iesu. 
„Die sehr dichte Korrespondenz der deutschen Hörer bietet die folgenden Informationen: Die Sendungen des Radio Vaticana sind ein echter Segen - gierig aufgenommene geistige Nahrung eine Notwendigkeit für die Deutschen - sie gibt ihnen das Gefühl, dass der Heilige Vater sie liebt und sich um sie sorgt - auch viele Protestanten, die sich sogar mit ihrem Pastor versammeln, folgen ihnen. “24

An einer anderen Stelle wird vermerkt, dass die deutschen Pfarrer die Nachrichten, die sie aus den Sendungen bezogen, zuweilen auch in ihren Predigten verbreiteten. So habe ein bayerischer, wegen antinationalen Verhaltens überwachter Priester (er hatte versucht, die Hakenkreuzfahne so zu hissen, dass sie kaum zu sehen war) anscheinend von der Kanzel herab eine möglicherweise von Radio Vaticana bezogene Meldung weitergegeben, wonach in Deutschland die Kruzifixe entfernt und in den Rhein oder auf den Düngerhaufen geworfen worden seien. Er habe auch nicht aufgehört, in seinen Predigten „Greuelnachrichten des Vatikansenders über Deutschland bekannt“ zu geben. ${ }^{25}$

\section{Nachgewiesene Fälle für den Empfang der Radiosendungen aus dem Vatikan}

Mit Ausbruch des Zweiten Weltkriegs stellte sich das Problem des Empfangs des vatikanischen Rundfunks für das nationalsozialistische Regime in doppelter Hinsicht: Es ging nun nicht mehr nur darum zu kontrollieren, was auf Deutsch übertragen und von der deutschen Reichsbevölkerung gehört wurde (Goebbels versuchte hier sofort geeignete Maßnahmen zu ergreifen), sondern auch ein Auge darauf zu halten, was die Anstalt in anderen Sprachen in die gegen Deutschland Krieg führenden Länder übertrug. Auch wenn der Heilige Stuhl sich für neutral erklärt hatte, fürchteten die Nationalsozialisten, er könne in den englisch- und spanischsprachigen Ländern eine antideutsche Propaganda betreiben, so dass sie schon bald beschlossen, alle Sendungen täglich abzuhören. ${ }^{26}$

Am 1. September 1939 erließ Goebbels die „Verordnung über außerordentliche Rundfunkmaßnahmen“, die den Empfang ausländischer Rundfunksender untersagte;

241937 waren in Deutschland 8167957 Radioapparate gemeldet, was 12,2\% der Gesamtbevölkerung (66840 000) entsprach. Ende 1939 lag die Zahl der Hörer in Deutschland bei 13711 325, Ende 1941 bei 1584311 und Ende 1942 bei 16113 466. Vgl. Développement de la radiodiffusion, in: Radiodiffusion, Juli 1937; Union Général de radiodiffusion, Rapport Général sur le développement et la situation actuelle de la Radiodiffusion internationale, Genève, Juni 1943, S. 4 f.

25 Witetschek, Die Kirchliche Lage (wie Anm. 21), Dok. 97, S. 320, und Dok. 99, S. 328 f.

26 In einem Dokument des Sonderdienstes Seehaus vom 28. März 1941 heißt es, wegen seiner „Hetzpropaganda“ werde der Vatikan „seit 15. Dezember 1940 vom Sonderdienst Seehaus systematisch beobachtet“; Auswärtiges Amt, Akten. Kirchenfragen, Bd. 2, in: Politisches Archiv, Berlin, S. 28. 
ein Verstoß dagegen wurde mit Haft geahndet und konnte auch die Todesstrafe nach sich ziehen. ${ }^{27}$ Nach Ansgar Diller mißachteten die Religionsvertreter beider Konfessionen häufig dieses Verbot, weil sie - die Katholiken beispielsweise aufgrund des Konkordats -, über gewisse Privilegien zu verfügen glaubten. ${ }^{28}$ Goebbels setzte sich nachhaltigst dafür ein, eine derartige Vorzugsstellung abzubauen. Der Sicherheitsdienst der SS unterwarf den Klerus einer systematischen Kontrolle. Aus einer Besprechung habe sich ergeben, dass die Geistlichen in erster Linie Radio Vaticana hörten. Sie „hätten sogar beschlossen, ,die Meldungen ausländischer Sender in vorsichtiger Form propagandistisch zu verwerten““. ${ }^{29}$ Im Frühjahr 1939 hatte Hitler den deutschen Rundfunkanstalten die Übertragung von Gottesdiensten verboten - gegen den Rat Goebbels', der ihn vergeblich umzustimmen versuchte. Im Einverständnis mit dem Auswärtigen Amt schlug der Propagandaminister am 18. September 1939 vor, religiöse Programme wieder zuzulassen, damit ausländische Sender nicht Fuß fassten, doch Hitler ließ sich nicht umstimmen. ${ }^{30}$

Bis heute ist es mir nicht gelungen, die Gesamtzahl der Geistlichen zu ermitteln, gegen die ein Prozess angestrengt wurde oder die man verurteilte, weil sie Radio Vaticana empfangen hatten. Aus meinen bisherigen Forschungen ergibt sich folgendes Bild: Die Vergehen, die mit der Lektüre von verbotenen Büchern oder Zeitungen oder dem untersagten Rundfunkempfang zusammenhingen, fielen nach der „Liste der tabellarischen Gliederungspunkte“ aus der biographischen und statistischen Erhebung zu den „Priestern unter Hitlers Terror“ unter die Kategorie „Staatsfeindliches Verhalten“. ${ }^{31}$ Die sicheren Fälle, die ich bisher namentlich zu identifizieren vermochte, belaufen sich auf sieben, worunter sich der Priester Friedrich Feyrer aus Markt Eisenstein, Diözese Budweis, im Protektorat Böhmen und Mähren befindet. Er war zunächst von der Gestapo wegen einer Stellungnahme zur Sonntagspflicht verwarnt worden, kam dann am 13. März 1942 zweifellos wegen Abhörens des Vatikansenders für zehn Tage in Schutzhaft und wurde von der Gestapo verhört. Das Sondergericht Eger verurteilte ihn zu drei Jahren Zuchthaus mit Verlust der bürgerlichen Ehrenrechte. Bis zum 19. April 1945 blieb er im Strafgefangenenlager „Elberegulierung“ in Griebo in Sachsen-Anhalt. ${ }^{32}$

Zwar vermag ein einziges Beispiel nicht zu verdeutlichen, wie weit das Phänomen verbreitet war, doch kann es immerhin bestätigen, dass Goebbels’ Verordnung, ohne es ausdrücklich zu sagen, auch Radio Vaticana einschloss, deren Empfang also fak-

27 Veröffentlicht in: Reichsgesetzblatt, 1939, Teil I, S. 1683.

28 Rundfunk in Deutschland, hg. von Hans Bausch, Bd. 2: Ansgar Diller, Rundfunkpolitik im Dritten Reich, München 1980, S. 312.

29 Ebd., S. 311.

30 Ebd., S. 313.

31 Priester unter Hitlers Terror. Eine biographische und statistische Erhebung, Bd. 1, bearb. von Ulrich von Hehl u.a., Paderborn u.a. 1996, S. $119 \mathrm{f}$.

32 Ebd., Bd. 2, S. 1718. 
tisch in Deutschland verboten war. Dieser Umstand hat zusammen mit dem Einsatz von Störsendern gegenüber ausländischen Rundfunkübertragungen zweifellos dazu beigetragen, die Zahl der Hörer der vatikanischen Rundfunkanstalt während des Krieges zu verringern. Gleichwohl wurden die deutschsprachigen Sendungen fortgesetzt, weil man sicher war, Personen zu erreichen, die die Meldungen weitergeben würden (wie der Fall des verhafteten Priesters beweist). Die vatikanischen Behörden wussten überdies, dass die Sendungen von deutscher Seite überwacht wurden und nutzten diesen Umstand als taktisches Instrument sowohl auf propagandistischer als auch auf diplomatischer Ebene.

\section{Die ersten Kriegsjahre: 1939-1941}

Am 21. September 1939 wandte sich Kardinal August Hlond, Primas von Polen und Erzbischof von Posen (Poznań) und Gnesen (Gniezno), über das vatikanische Studio an die polnische Bevölkerung; seine Rede wurde am 2./3. Oktober im „Osservatore Romano“ veröffentlicht. ${ }^{33}$ Die Vergeltungsmaßnahmen der Gestapo beschrieb Monsignor Carl Maria Splett, Bischof von Danzig und apostolischer Administrator der Diözese Kulm (Chetmno), in einem Brief vom 14. Januar 1940 an Pius XII. ${ }^{34}$ Die Nationalsozialisten, so Splett, hätten von Ende Oktober bis Ende November schrecklich gewütet. Nach Aussage der Gestapo habe Kardinal Hlond die polnische Bevölkerung zum Widerstand aufgerufen und die Gläubigen aufgefordert, sich um den eigenen Klerus zu scharen. Man habe ihm, Splett, zu verstehen gegeben, dass daraufhin „unzählige Geistliche und Lehrer entweder verhaftet worden, erschossen worden, auf furchtbarste Art zu Tode gequält worden oder auch nach dem weiten Osten verschickt worden“ seien. ${ }^{35}$ Zum 21. Dezember 1939 verfasste Kardinal Hlond einen detaillierten Bericht, in dem er die Brutalität darstellte, mit der die deutschen Besatzer in seinen beiden Erzdiözesen gegen die katholische Kirche vorgegangen waren. Zwei Tage später berief der Papst eine Sitzung der Kongregation für die außerordentlichen kirchlichen Angelegenheiten ein, um über mögliche Gegenmaßnahmen zu beraten. ${ }^{36}$ Hieraus ging die Entscheidung des Papstes vom 19. Januar 1940 hervor, sich der vatikanischen Rundfunkanstalt zu bedienen. ${ }^{37}$ Folgender Text wurde in deutscher Sprache übertragen:

33 Vgl. die Radioansprache von Kardinal Hlond, in: L'Osservatore Romano, 2./3.10.1939, S. 2. Vgl. auch Alberto Monticone, L'offensiva radiofonica nazista contro il card. Hlond nel 1940, in: Seminare. Poszukiwania naukowe 4 (2014), S. 121-141, auch in: Revue d'Histoire Ecclesiastique 3-4 (2015), S. $783-814$.

34 ADSS, Bd. 3a, Dok. 96, S. 194-197.

35 Ebd., S. 195.

36 Ebd., Dok. 74, S. 162.

37 Notiz Mons. Montinis vom 19. Januar 1940. Ex Audientia SS.mi., ebd., Dok. 102, S. 204: „Radio Vaticana mit einigen Fakten für die deutsche Sendung über die Lage der Kirche in Polen versorgen.“. 


\begin{abstract}
„Many Polish families in various towns are completely broken up. The father is in prison, the son and daughter have been sent to hard labour in Germany and the mother has been driven from her home and deported to central Poland. Since last November, the Poles have been systematically driven out of Posen, Pomerania and Silesia, and sent to the so-called Protectorate. We have no words for the method employed. Poles have to leave their house within a few minutes are brought, without any belongings, into the concentration camps, whence they are transported in closed trains to Central Poland. They are then ,freed', which means that these poor people are left to their fate, without homes, clothes and money. They are turned into beggars and left to their misery. Some of these journeys have been made in open trains in spite of the cold, others in closed goods trains - as for instance in the transport of Poles from Bromberg to Radow, when, for three days no one was allowed to leave the goods wagons. “38
\end{abstract}

Tatsächlich strahlte Radio Vaticana diesen Bericht mit einigen Varianten auch in anderen Sprachen aus. ${ }^{39}$ Bereits am 19. Januar hatte sich Staatssekretär Ernst von Weizsäcker beim Nuntius über eine tags zuvor ausgestrahlte Übertragung zur wirtschaftlichen Situation in den polnischen Gebieten beklagt. ${ }^{40}$ Im Februar drückte Orsenigo während einer Audienz bei Ribbentrop sein Missfallen darüber aus, dass eine große Zahl von katholischen Priestern verhaftet worden sei. Der Minister notierte, er sei auf diese Beobachtung nicht eingegangen, habe die Rede vielmehr auf die wenig freundlichen Sendungen der vatikanischen Rundfunkanstalt über Polen gebracht. Der Nuntius erklärte, dass in dieser Angelegenheit der Botschafter von Bergen bereits aktiv geworden sei und sich Derartiges nicht mehr wiederholen würde. In der Tat war der Beauftragte der deutschen Botschaft beim Heiligen Stuhl, Menshausen, am 27. Januar beim Staatssekretariat vorstellig geworden, um sich $\mathrm{zu}$ beschweren. ${ }^{41}$

Das Staatssekretariat antwortete in der Regel, dass es nicht verantwortlich sei und keine Kenntnis davon habe, was Radio Vaticana übertragen würde. Nun wissen wir, dass zumindest im vorliegenden Fall der Papst selbst angeordnet hatte, Sendungen über Polen auszustrahlen. Ein Vermerk Monsignor Tardinis vom 30. Januar unterrichtet uns allerdings auch darüber, dass Pius XII. aufgrund der Proteste des deutschen Diplomaten verfügt habe, sie wieder einzustellen. ${ }^{42}$ Eine weitere Überlegung schließt sich hier mit Blick auf die chronologische Abfolge an: Aus dem Eingangs-

38 BBC Written Archives, Daily Digest of Foreign Broadcasts. Vatican City, in deutscher Sprache für Deutschland, 23. Januar 1940, 19.30 Uhr. Alle hier zitierten Texte aus dem Daily Digest der BBC sind im Original auf Englisch verfasst.

39 Am 21./22. Januar auf Englisch für Nordamerika, am 24. Januar auf Französisch und Spanisch und am 26. Januar auf Spanisch für Lateinamerika.

40 Der Notenwechsel zwischen dem Heiligen Stuhl und der deutschen Reichsregierung, Bd. 3: Der Notenwechsel und die Demarchen des Nuntius Orsenigo 1933-1945, bearb. von Dieter Albrecht, Mainz 1980, Dok. 475.

41 ADSS, Bd. 3a, Dok. 108, S. 208 f.

42 Ebd., Vermerk vom 31. 1. 1940: „Der Heilige Vater teilt mir mit, er habe verfügt, momentan mit den Sendungen auszusetzen. Ich antworte mit dem Wunsch, dass ... sie bald wieder aufgenommen würden. Seine Heiligkeit lächelt zustimmend.“. 
protokoll ergibt sich nicht, ob Spletts Brief, der vom 14. Januar datiert, vor oder nach der Entscheidung des Papstes vom 19. Januar eintraf. Sollte er vorher angekommen sein, wäre zweifellos interessant festzuhalten, dass Pius XII. Spletts Informationen über die Repressalien im Zusammenhang mit der Übertragung von Hlonds Rede nicht berücksichtigt hat.

Von April bis Juni 1940 kam es zu einem umfangreichen Notenwechsel zwischen von Weizsäcker, Orsenigo, dem deutschen Botschafter beim Heiligen Stuhl von Bergen, dessen Chargé d'Affaire und dem Staatssekretariat. An ihm zeigt sich, wie das deutsche Außenministerium die Informationen über die vatikanische Sendeanstalt dazu nutzte, um die Verfolgung des Klerus und der Katholiken in den besetzten Gebieten zu rechtfertigen und gegenüber dem apostolischen Nuntius, letztlich also gegenüber dem Heiligen Stuhl, als Druckmittel einzusetzen. Wenn Radio Vaticana die antinationalsozialistischen Sendungen einstellte, hieß es, würden auch die Verfolgungen aufhören. ${ }^{43}$ Angesichts der Befürchtungen und tiefen Verärgerung, die Radio Vaticana bei verschiedenen deutschen Ministerien hervorrief, sind Zweifel am Ursache-Wirkungs-Verhältnis zwischen den antinationalsozialistischen Inhalten der Rundfunksendungen und den Repressalien gegenüber dem polnischen Klerus erlaubt. Vermutlich lag auch bei der nationalsozialistischen Diplomatie ein instrumenteller Gebrauch dieser Übertragungen vor, dienten sie doch dazu, Gewalttaten, die auf jeden Fall begangen worden wären, post festum zu rechtfertigen. Das Amt des Generalgouverneurs für die besetzten polnischen Gebiete hatte beispielsweise „[mit] Rücksicht auf die deutschfeindliche Haltung der Presse und des Rundfunks des Vatikans“ im Mai angeordnet, „dass katholischen Priestern und Ordensangehörigen die Ausreise nicht zu gestatten sei“. Am 13. Juli schrieb Orsenigo an Maglione, der Außenminister, bei dem er vorstellig geworden sei, habe ihm gesagt, die polnischen Priester und Ordensangehörigen dürften Polen noch nicht verlassen, während die Ausländer, die sich im Land aufhielten, einen Antrag stellen könnten. Der Grund für die Aufrechterhaltung dieser Beschränkungen sei darin zu suchen, dass „Radio Vaticana nach Ansicht der deutschen Regierung den rechten Ton noch nicht getroffen habe, so dass man sich dort nicht beklagen dürfe“ “. ${ }^{4}$

Am 22. April 1940 wandte sich Pius XII. mit einem Schreiben an den Berliner Bischof Konrad von Preysing, worin er ihn im wesentlichen fragte, ob es angebracht sei, dass Radio Vaticana über die Lage der Kirche in Deutschland berichte: ${ }^{45}$

„Zwei Fragen möchten Wir heute Unsererseits dir, ehrwürdiger Bruder, vorlegen. Die erste betrifft die Berichte des übrigens für gewöhnlich nicht amtlichen Vatikansenders über die Lage der katholischen Kirche in Deutschland. Die Berichte sind gegeben worden aus der Erwägung, dass ein vollständiges Schweigen des Hl. Stuhles in der Oeffentlichkeit geeignet gewesen wäre,

43 Albrecht (vgl. Anm. 40), passim.

44 ADSS, Bd. 3a, Dok. 152, Annexe, S. 256; Dok. 172, S. 272 f.

45 ADSS, Bd. 2, Dok. 45, S. 138-142. 
die deutschen Katholiken zu entmutigen und ausserhalb Deutschlands das Missverständnis zu fördern, als ob die kirchlichen Dinge in Deutschland eigentlich ziemlich normal stünden, jedenfalls sich gebessert hätten. Dieser mit Geschick und immer noch mit Erfolg betriebenen Tarnung haben die mit der Sendung Betrauten aber gerade begegnen wollen. Andererseits kommen, auch von bischöflichen Stellen, Klagen, ja Notrufe wegen der vom Gegner für die Berichte des Vatikansenders verhängten Repressalien. Wir möchten gewiss den deutschen Katholiken keine unnötigen Opfer auferlegen, wo sie um ihres Glaubens willen schon so bedrängt sind. Wir haben deshalb jene Berichte vorerst einstellen lassen, bis Wir das Für und Wider mit mehr Sicherheit abschätzen können. Wir wären dir sehr verbunden, wenn du Uns dein geschätzes Urteil und deine Erfahrung in der Angelegenheit mitteilen wollest.“

Von Preysing antwortete am 1. Mai:

„Der Heilige Stuhl kann wohl nicht darauf verzichten, kirchliche Ereignisse, auch solche, die sich in Deutschland vollziehen, zum Gegenstand der Besprechung vor der Weltöffentlichkeit zu machen. Abraten möchte ich, Dokumente des internen Verkehrs, Eingaben, Proteste als solche $\mathrm{zu}$ veröffentlichen. Aber Mitteilungen über kirchenfeindliche Massnahmen, besonders wenn sie notorisch sind, Enteignungen, Aufhebung von Vereinen, Beschlagnahme der Gelder katholischer Institutionen nach dem Gesetz über kommunistische Umtriebe etc. können m[einer] $\mathrm{M}\left[\right.$ einung] $\mathrm{n}[\mathrm{ach}]$ unbedenklich besprochen werden. ${ }^{“ 46}$

Dem Ratschlag entsprechend wurden die Sendungen fortgeführt. Pius XII. versuchte nun sowohl über die Kommunikationsmedien als auch unter Nutzung der diplomatischen Kanäle zu sondieren, wie sich die Unabhängigkeit des Heiligen Stuhls wahren ließ, ohne auf die Verteidigung der Rechte der Katholiken verzichten zu müssen.

In der ersten Hälfte des Jahres 1941 eskalierte die zunehmend angespannte Diskussion zwischen dem Heiligen Stuhl und der deutschen Botschaft über die vatikanischen Rundfunksendungen. Im vorliegenden Zusammenhang, in dem es über die Beziehungen zwischen dem Vatikan und dem Dritten Reich geht, gilt es hervorzuheben, dass die Rundfunkanstalt in dieser Phase die Lage der katholischen Kirche in den von Deutschland besetzten Territorien anklagte und vor allem in den nach Nord- und Südamerika ausgestrahlten französisch-, englisch- und spanischsprachigen Sendungen Nationalsozialismus und Kommunismus faktisch auf die gleiche Stufe stellte. Ein erneuter Protest Menshausens beim Staatssekretariat veranlasste Pius XII. im April 1941 schließlich, die Berichte über Deutschland auszusetzen, und nach weiteren Stellungnahmen der italienischen Botschaft zugunsten des Verbündeten ordnete er an, die Sendungen einer Vorzensur zu unterziehen. ${ }^{47}$

46 Ebd., Anm. 7, S. 141.

47 Vgl. dazu Perin, La radio del papa (wie Anm. 6), S. $101 \mathrm{f}$. 


\section{Die Rundfunksendungen unter der Vorzensur}

Bei Kriegsausbruch vertrat der deutsche Episkopat zunächst eine Linie, die im Großen und Ganzen der traditionellen katholischen Lehre über den Krieg und die Gehorsamspflicht gegenüber der legitimen Staatsgewalt entsprach, auf die sich die Päpste seit der zweiten Hälfte des 19. Jahrhunderts verstärkt beriefen: Er versicherte dem Regime seine Loyalität, forderte die Gläubigen auf, die Vaterlandspflichten zu erfüllen und drückte seine Hoffnung auf einen Sieg aus. ${ }^{48}$ Je länger der Krieg allerdings dauerte und in den Methoden, Massakern und Deportationen sein ganzes Grauen offenbarte, machten sich unter den Bischöfen Unsicherheit und Spannungen breit; sie führten bald schon zu Spaltungen, die vor dem Zusammenbruch des Dritten Reiches nicht mehr überwunden wurden. Vereinfachend lassen sich zwei Orientierungen benennen. Eine Mehrheit um Kardinal Adolf Bertram, Bischof von Breslau und Präsident der Fuldaer Bischofskonferenz, tendierte zum Kompromiss mit dem Nationalsozialismus und lehnte es ab, öffentlichen Dissens zu äußern, eine zweite Gruppe um den Berliner Erzbischof Konrad von Preysing zeigte sich eher bereit, das Regime offen zu kritisieren. Der Nuntius Orsenigo hielt es für angebracht, öffentliche Erklärungen gegen Hitler und dessen Regierung zu vermeiden, da sie als staatsfeindlich aufgefasst worden wären: Man könne den Nationalsozialismus nicht kritisieren, ohne als Vaterlandsverräter gebrandmarkt zu werden. ${ }^{49}$ Eine den Gehorsam gegenüber den politischen Autoritäten und das Gebot der nationalen Solidarität im Krieg unberührt lassende Unterscheidung zwischen Vaterland, Staat und Partei, die dem Episkopat als Richtschnur hätte dienen sollen, wurde vom Regime nicht akzeptiert. ${ }^{50}$ Wachsende Umsicht folgte aus dieser tief empfundenen Notwendigkeit, der für legitim gehaltenen Regierung treu zu bleiben und zu gehorchen in dem Versuch zu retten, was noch zu retten war. Sie ergab sich aber auch aus Furcht, Zielscheibe einer neuen Dolchstoßlegende zu werden, wonach die defätistische Opposition im eigenen Land die Niederlage im Ersten Weltkrieg heraufbeschworen habe und auf die die nationalsozialistische Propaganda vor und während des Zweiten Weltkrieges immer wieder abhob.

Kardinal Faulhaber wies darauf ganz offen in der Konferenz der bayerischen Bischöfe vom 30. und 31. März 1943 hin:

„Viel Stimmung oder Mißstimmung wird auch in katholischen Kreisen gemacht durch das Schlagwort: Warum treten die Bischöfe nicht auf? Warum reden die Bischöfe nicht? Die Chance für die katholische Kirche sei niemals größer gewesen. Responsum: Wir könnten den Gegnern der Kirche keinen größeren Gefallen tun, als jetzt große Kanonen aufzufahren. Jetzt, wo man in

48 Vgl. die Analyse von Miccoli (wie Anm. 5), S. 181-212.

49 In diesem Sinne schrieb Orsenigo am 17. Januar 1941 an Maglione, in: ADSS, Bd. 4, Dok. 238, S. 347-351, hier S. 350.

50 Miccoli (wie Anm. 5), S. 204. 
Schwierigkeiten steckt, würde man sofort die Geschichte vom Dolchstoß wieder aufleben lassen. Mir scheint, man wartet darauf. “51

Der Papst seinerseits fürchtete, dass mögliche Äußerungen von ihm negative Auswirkungen auf die Lage der deutschen Katholiken haben könnten, und überließ es deshalb den Bischöfen, die Stimme zu erheben, wenn sie es für nötig hielten; seiner Unterstützung sollten sie auf jeden Fall sicher sein..$^{52}$

Eine Analyse der deutschsprachigen Sendungen legt den Schluss nahe, dass Pius XII. in der vatikanischen Rundfunkanstalt eines der Instrumente für seine strategischen Pläne gefunden hatte. Im Verlauf des Jahres 1942 übertrug Radio Vaticana in deutscher Sprache zahlreiche Hirtenbriefe des europäischen Episkopats, deren Botschaft insgesamt lautete, dass die Kirche das deutsche Volk nicht aufgegeben habe. Die gleichzeitige Ablehnung der rassistischen und freiheitsfeindlichen Theorien, so betonte man, stehe in vollständigem Einklang mit der Treue zum Vaterland, das damit nicht mit dem nationalsozialistischen Deutschland, d.h. mit Hitlers Partei und Ideologie identisch gesetzt wurde. Nach diesen Kriterien erfolgte möglicherweise die Auswahl der Hirtenbriefe, die schließlich übertragen wurden.

Das Jahr 1942 begann für die deutschen Radiohörer mit zwei Sendungen über den Mut. ${ }^{53}$ In der zweiten wurde die Neujahrsansprache des Freiburger Erzbischofs Monsignor Gröber übertragen, worin dieser den Wunsch ausdrückte: „May it, for our Nation and Fatherland, not be night, but a night during which the eternal stars continue to shine and after which the glowing dawn of a happier day is beckoning. “54 In der Anrufung von „Nation und Vaterland“ sprach der Erzbischof vorsichtigerweise nicht ausdrücklich von Sieg und Frieden, sondern griff auf die Metapher von Licht und Finsternis zurück, mit der er die Hoffnung auf glücklichere Tage ausdrückte.

Mit dem Hirtenbrief vom 27. Januar, der im Diözesanblatt vom 5. Februar erschien und auch an den Papst geschickt wurde, wurde ein weiterer Text Gröbers von Radio Vaticana in deutscher Sprache verlesen. ${ }^{55}$ Der Abhörer der BBC notierte folgende Stelle:

51 Volk (wie Anm. 13), S. 983.

52 Vgl. auch hier Miccoli (wie Anm. 5), der eine Reihe von Briefen Pius' XII. an die deutschen Bischöfe auflistet, worin er seine Position erklärt; ebd., S. 207 f. und Anm. 317.

53 BBC Written Archives, Daily Digest of Foreign Broadcasts. Vatican City, in deutscher Sprache für Deutschland, 13. und 14. Januar 1942, 19.45 Uhr.

54 Die Sendung erschien auch gedruckt in der englischen katholischen Zeitung „Tablet“: News, Notes and Texts. Germany: Dark Times Ahead, in: The Tablet, 24.1.1942, S. 47.

55 BBC Written Archives, Daily Digest of Foreign Broadcasts. Vatican City, in deutscher Sprache für Deutschland, 10. März 1942, 19.45 Uhr. Pacelli antwortete mit einem Brief vom 1. März 1942 auf Gröbers Huldigung; vgl. ADSS, Bd. 2, Dok. 84, S. 255-260. 


\begin{abstract}
„At no time we injure the interests of the Fatherland, or impair them. We recognise in the State only the tool of Him who creates eternal order. The Church did not fail when asked to do its duty for King and Country. There came the war of 1914. The whole of Germany at that time was full of religious fervour. When the collapse came, the Church did everything in its power to inspire the people for the last effort. The Archbishop says the German defeat made him weep. After the war, the fight against Rome flared up again and some people ascribed the guilt for the war and the Treaty of Versailles to the Church.“
\end{abstract}

Gröber wollte damit die Kirche gegen den Vorwurf in Schutz nehmen, dass sie das deutsche Volk im Krieg nicht unterstütze und einem der Dolchstoßlegende zugrundeliegenden Defätismus huldige. Gröber, der zu den Bischöfen gehörte, die am häufigsten von Radio Vaticana zitiert wurden, hatte sich anfänglich dem Nationalsozialismus angeschlossen, war dann aber uneingeschränkt dafür eingetreten, die religiöse Verfolgung in Deutschland öffentlich anzuprangern. Er war jedoch auch ein Patriot, der versuchte, die Moral der Soldaten und ihrer Familien zu heben. ${ }^{56}$ Einige Monate nach der Übergabe seines Hirtenbriefes an den Papst und dessen Ausstrahlung durch die vatikanische Rundfunkanstalt verfasste Gröber für Pius XII. einen langen Bericht über die Lage in seiner Diözese, worin er ihm mitteilte, dass die Nazionalsozialisten von der Theorie zur Praxis übergegangen seien und auf die Vernichtung der Juden zielten, von denen bisher mindestens 220000 umgebracht worden seien. ${ }^{57}$ Diese Informationen wurden allerdings nicht übertragen und waren möglicherweise gar nicht in den Besitz der Redaktion gelangt.

Im April wurde der Hirtenbrief des Münsteraner Bischofs Clemens August von Galen über die religiöse Erziehung verlesen, der sich vor allem an die katholischen Eltern richtete und sie an ihre heilige Pflicht erinnerte, ihre Kinder christlich zu erziehen, insbesondere in einer Zeit, in der die Schule diese Aufgabe nicht mehr erfüllte. ${ }^{58}$ Während sich die deutschen Bischöfe, die sich Anfang des Monats in Bonn versammelt hatten, im Rahmen der Feiern zum Bischofsjubiläum des Papstes am 28. Juni 1942 mit einem Hirtenschreiben an die Gläubigen wandten und sie aufriefen, mit einer geistigen Pilgerschaft nach Rom ihren Glauben und ihren Gehorsam gegenüber dem Papst zu bezeugen. ${ }^{59}$ Es folgte eine Lobrede auf Pius XII. mit Blick auf seine Nuntiatur in Deutschland:

„Pius XII spent more than a half his years as a Bishop in our Fatherland, not grudgingly like many, a foreigner who consider his stay beyond the Alps as a sort of exile, not as a cold observer who notices, registers, and draws conclusions; no, he looked about him with his eyes of love,

56 Vgl. Miccoli (wie Anm. 5), S. 185 und die in Anm. 228 zitierten Werke.

57 Der Brief datiert vom 14. Juni 1942 und wird ebd., S. 7, zitiert.

58 BBC Written Archives, Daily Digest of Foreign Broadcasts. Vatican City, in deutscher Sprache für Deutschland, 13. April 1942, 20.45 Uhr.

59 Akten deutscher Bischöfe über die Lage der Kirche 1933-1945, Bd. 5: 1940-1942, bearb. von Ludwig Volk, Mainz 1983, Dok. 769, S. 754-761, hier S. 755. 
being greatly interested in the heartbeats of this Nation, which is alien in blood but near in faith ... Archbishop Pacelli’s mission in Germany was a mission of peace and of spiritual reconstruction." 60

Pacelli wurde nicht nur als Förderer des Friedens gefeiert, vor allem würdigte man seine Sympathie für das deutsche Volk, die gleichsam als Beweis dafür stand, dass der Papst die Niederlage Deutschlands nicht wünschen konnte.

Anlässlich des fünfzigsten Jahrestages seiner Priesterweihe und seines Silberjubiläums als Münchner Erzbischof verfasste Kardinal Faulhaber einen Hirtenbrief, der von Radio Vaticana übertragen und vom „Tablet“ abgedruckt wurde; der Kardinal hob darin unter anderem hervor, wie bereitwillig die Seminaristen seiner Diözese der Sache des Vaterlandes dienten. ${ }^{61}$ Am 18. September verlas die vatikanische Radioanstalt den gemeinsamen Hirtenbrief der deutschen Bischöfe, die sich vom 18. bis zum 20. August 1942 in Fulda zum Plenum versammelt hatten. ${ }^{62}$ Auch dieses Mal konnte die englische katholische Wochenzeitung dank der Sendung umfassend darüber berichten. ${ }^{63}$ In ihrer Stellungnahme betonten die Bischöfe - wie schon in ihren jeweiligen Diözesanbriefen - ihre Nähe zu den Soldaten, die mit „,soviel Heldenmut“ für ihr Vaterland kämpften und starben, und bekräftigten damit, dass es eine religiöse, das ewige Heil verheißende Pflicht sei, den Befehlen der Autoritäten Folge zu leisten; ${ }^{64}$ auf diese mittlerweile klassisch gewordene Linie der katholischen Lehre hatte auch Pius XII. bei verschiedenen Gelegenheiten zurückgegriffen. Ferner gedachten sie der Priester, die sich in der Heimat unter Aufbringung ihrer letzten Kraft aufopferungsvoll um ihre Schutzbefohlenen kümmerten, an die Ordensfrauen und Mütter, an die Mitbrüder und Mitschwestern, die ihre Söhne, Väter und Ehemänner verloren hatten. Schließlich riefen sie den „allmächtigen und allgütigen Gott“ an, er möge dem Land und der Welt Frieden schenken. Sie unterließen es allerdings nicht hinzuzufügen: „During these days, we have been occupied with serious questions and immediate anxieties, and we considered it our sacred duty to turn to the competent authorities. However, in this time of difficulties of war (kriegsschweren) [sic], we do not think it appropriate to give further details in this episcopal letter.“

60 BBC Written Archives, Daily Digest of Foreign Broadcasts. Vatican City, in deutscher Sprache für Deutschland, 2. Juli 1942, 21.45 Uhr.

61 News, Notes and Texts. Germany, in: The Tablet, 8.8.1942, S. 67.

62 Vgl. den gesamten Text des Hirtenbriefes in Volk (wie Anm. 59), Dok. 788, S. 913-918.

63 BBC Written Archives, Daily Digest of Foreign Broadcasts. Vatican City, in deutscher Sprache für Deutschland, 18. September 1942, 20.45 Uhr; News, Notes and Texts. Germany, in: The Tablet, 26.9.1942, S. 155.

64 Vgl. beispielsweise seine Ansprache an die Führung der Azione cattolica italiana vom September 1940, die Daniele Menozzi, Chiesa, pace e guerra nel Novecento. Verso una delegittimazione religiosa dei conflitti, Bologna 2008, S. 155, analysiert hat. 
Es ist nicht klar, worauf sich die Bischöfe genau bezogen, vielleicht auf die jüngst erfolgte Verhaftung von drei katholischen Priestern, einem lutherischen Pfarrer und 18 Laien, die im darauffolgenden Jahr wegen Defätismus zum Tode verurteilt wurden. ${ }^{65}$ Auf jeden Fall würde die Kirche, hieß es weiter, mutig fortfahren, die Unwahrheit und den Irrtum, darunter den Liberalismus und den widerchristlichen Kollektivismus, zu bekämpfen sowie für die Gewissensfreiheit, die menschliche Würde und die Freiheit in der Ausübung der von Gott unmittelbar dem Menschen gegebenen Rechte einzutreten.

Die Antwort des Papstes auf diesen gemeinsamen Hirtenbrief und auf das Schreiben, das am 25. Oktober verfasst und ihm anlässlich seines Priesterjubiläums am 8. Dezember zugesandt worden war, wurde einige Monate später von Radio Vaticana ausgestrahlt. ${ }^{66}$ Interessant ist der im Dezember 1942 erschienene Kommentar des „Tablet“. Im Laufe eines Jahres hätten die deutschen Bischöfe vier gemeinsame Hirtenbriefe verfasst. Der erste sei am Palmsonntag in allen deutschen Kirchen verlesen, der zweite anlässlich des Priesterjubiläums Pius’ XII. veröffentlicht worden. Den dritten hatte das Blatt in seiner Ausgabe vom 26. September auf der Grundlage der Übertragung von Radio Vaticana zusammengefasst. ${ }^{67}$ Er wäre völlig unbekannt geblieben, hieß es, wäre der Radiosender nicht auf ihn eingegangen. Auch wenn dem Leitartikler dabei schien, dass der ursprünglich von den Bischöfen formulierte Text sehr viel länger gewesen sei und Radio Vaticana mit seiner Zusammenfassung den Eindruck erweckt habe, der deutsche Episkopat wolle sich der Regierung gegenüber konziliant zeigen, schloss er die Möglichkeit aus, es habe die bewusste Absicht bestanden, dem Reich eine Art Verständnisbereitschaft zu signalisieren, vermutete vielmehr, dass die Nationalsozialisten die Verbreitung des vollständigen Textes verhindert hätten. Tatsächlich zeigt ein Vergleich zwischen der Mitschrift der BBC und dem in den „Akten deutscher Bischöfe“ veröffentlichten Original, dass es keine wesentlichen Abweichungen gibt, woraus geschlossen werden kann, dass die Sendeanstalt keine Kürzungen aus strategischen Gründen vorgenommen hatte und die Bischöfe ursprünglich keine schärferen Anklagen vorgebracht hatten.

Mit der Predigt, die der Fuldaer Bischof Johannes Schmidt am 24. November 1942 anlässlich des Christkönigsfestes gehalten hatte, übertrug Radio Vaticana am 21. Dezember - vor den Kommentaren zur Weihnachtsansprache Pius' XII. - zum

65 Vgl. Testimoni di Cristo. I martiri tedeschi sotto il nazismo, hg. von Helmut Moll, Cinisello Balsamo 2007, S. 203-213.

66 ADSS, Bd. 2, Dok. 93, S. 280-284; BBC Written Archives, Daily Digest of Foreign Broadcasts. Vatican City, in deutscher Sprache für Deutschland, 27. Mai 1943, 21.45 Uhr.

67 News, Notes and Texts. Germany: Four Fulda Pastorals, in: The Tablet, 19.12.1942, S. 300 f. Der vierte Hirtenbrief datiert vom 22. März 1942, über den „The Tablet“ am 20.6.1942, S. 306 f. in: The battle raging in Germany, berichtet. 
letzten Mal in diesem Jahr die Stellungnahme eines deutschen Prälaten. ${ }^{68}$ Nach dem Urteil des Ansagers handelte es sich dabei um eine jener hervorragenden Reden, die man vom deutschen Episkopat zu hören gewohnt war. Schmidt beklagte, dass in Deutschland die Propaganda gegen den christlichen Glauben noch so weit verbreitet sei: „A good many in our Fatherland reject Christ as the universal King, and nothing is so antagonistic to them as the unity of all men in His Kingdom. They want to split up mankind, and hold that varying national and racial characteristics preclude spiritual unity and community."

Nur die unbedingt-absolute Natur der Wahrheit, fuhr der Bischof fort, könne diese Gefahr bannen; deshalb sei es auch nicht möglich, dass jeder Staat seine eigene Wahrheit vertrete, die zu jener in Widerspruch stehe.

\section{Das Wort dem deutschen Episkopat}

Schon die Niederlage der Achsenmächte in Nordafrika im Jahr 1942 bildete einen tiefen Einschnitt. 1943 kam es dann zu weiteren Einbrüchen auf den Schlachtfeldern, die sich zwangsläufig auf der politischen Ebene auswirkten; die deutsche Kapitulation in Stalingrad stellte hier einen entscheidenden Wendepunkt dar.

Radio Vaticana leitete seine deutschsprachigen Sendungen im Jahr 1943 mit zwei Radiogesprächen ein. Im ersten wurden die Hörer mit Blick auf die päpstliche Weihnachtsbotschaft aufgefordert, für den Frieden zu beten, im zweiten wurde betont, dass die katholische Ordnung die einzig wahre sei, weil sie sich auf das Wort Gottes gründe: Es gebe keine kollektive oder individuelle Lösung, sondern nur eine universelle in Christus. ${ }^{69}$ Die deutschen Übertragungen wandten sich weiterhin Themen wie der neuen Ordnung, der Einheit der Völker, der Freiheit zu, erwähnten dabei allerdings nicht ausdrücklich Deutschland, sondern zogen geschichtliche oder literarische Beispiele heran, mit denen sie auf Situationen in der Gegenwart anspielten. ${ }^{70} \mathrm{Am}$ 11. Januar beispielsweise wurde eine Radiokonferenz über den Auszug des jüdischen Volkes aus Ägypten übertragen, den der Sprecher nicht als Ausdruck von Feigheit deutete, sondern als Anstoß für die Kirche betrachtete, nach einem Weg zu suchen, mit dem sich die Verfolgungen vermeiden ließen. Unmittelbar danach wurde die Stelle

68 BBC Written Archives, Daily Digest of Foreign Broadcasts. Vatican City, in deutscher Sprache für Deutschland, 21. Dezember 1942, 20.45 Uhr; News, Notes and Texts. Germany, in: The Tablet, 9.1.1943, S. 18 .

69 Ebd., 4. Januar 1943, 20.45 Uhr.

70 Das Thema von Antisemitismus und Shoah in den deutschsprachigen Sendungen bleibt hier ausgeklammert, es ist spezifischer Gegenstand von zwei Artikeln in Perin, Vatican Radio (wie Anm. 6) und dies., Vatican Radio and Anti-Semitism during the Second World War, in: „The Tragic Couple“. Encounters Between Jews and Jesuits, hg. von James Bernauer/Robert A. Maryks, Leiden-Boston 2014, S. 247-267. 
aus dem Brief an die Korinther erläutert, wo der Apostel Paulus seine Mitbrüder aufforderte, keine Unstimmigkeiten untereinander aufkommen zu lassen. ${ }^{71}$ Eine weitere Sendung war der staatlichen Macht gewidmet. Jene Staaten, die jegliche Beziehung zur überweltlichen Autorität abgebrochen hätten, würden trotz ihrer zeitweiligen Erfolge letztlich vergehen, wurden die Hörer ermahnt und daran erinnert, dass der Staat dem Heil des Menschen und nicht - umgekehrt - der Mensch dem Heil des Staates diene..$^{72}$

$\mathrm{Zu}$ einer ausdrücklichen Bezugnahme auf die aktuelle Lage kam es unter diesen zahlreichen Sendungen religiösen Inhalts erstmals am 3. März 1943, als eine Rede verlesen wurde, mit der der Kölner Erzbischof Josef Frings den Gläubigen den Fall Stalingrads zu erklären versuchte: „We are deeply moved and humbled before the heroism of the fighters of Stalingrad. ... We have understood God's call. The time has come to turn to Him in a ,storm of prayer`. May He protect our soldiers and our Fatherland. “73

Der Erzbischof gebot besondere Fürbitten und Gottesdienste für die deutsche Nation und ordnete an, in allen Kirchen eine Trauerfeier für die in Stalingrad gefallenen Soldaten abzuhalten.

Am 22. März 1943 wurden einige Punkte aus einem Hirtenbrief verlesen, den der deutsche Episkopat im Vorjahr gemeinsam verfasst hatte, der aber im Gegensatz $\mathrm{zu}$ anderen nicht von Radio Vaticana ausgestrahlt worden war. ${ }^{74}$ Am 10. Dezember 1941 hatten die Bischöfe der nationalsozialistischen Regierung ein Memorandum überreicht, in dem die Verfolgung der deutschen Katholiken dokumentiert und die Achtung der für jeden Menschen unveräußerlichen natürlichen Rechte eingefordert wurde. Die nationalsozialistische Regierung reagierte nicht, doch der Erwartungsdruck seitens der katholischen Gläubigen, eine klare öffentliche Stellungnahme abzugeben, und das in dieselbe Richtung gehende Drängen Pius' XII. veranlassten den Episkopat, unter dem 22. März 1942 einen gemeinsamen Hirtenbrief abzufassen, von dem Radio Vaticana dann auf den Tag genau ein Jahr später eine Zusammenfassung sendete. ${ }^{75}$ Anders als vom Ansager einleitend behauptet, war der Text zum Zeitpunkt seiner Entstehung nicht in allen deutschen Kirchen verlesen worden. Vor allem Kardinal Bertram lehnte den ursprünglichen Inhalt des Hirtenbriefes ab, weil er die Kirche

71 Ebd., 11. Januar 1943, 20.45 Uhr. Der zitierte Passus findet sich in Cor. I,10.

72 Ebd., 25. Februar 1943, 20.45 Uhr.

73 Ebd., 3. März 1943, 20.45 Uhr.

74 Dass Radio Vaticana den gemeinsamen Hirtenbrief vom 22. März 1942 nicht ausstrahlte, wird dadurch bestätigt, dass das Blatt „The Tablet“, das ihn veröffentlichte, den Text aus einer anderen Quelle bezog; er wurde dem Würzburger Bischof zugeschrieben, einem der wenigen im übrigen, die die ursprüngliche Fassung vor Bertrams Eingriff gelesen hatten. Vgl. The battle raging in Germany, in: The Tablet, 20.6.1942, S. $306 \mathrm{f}$.

75 BBC Written Archives, Daily Digest of Foreign Broadcasts. Vatican City, in deutscher Sprache für Deutschland, 22. März 1943, 20.45 Uhr. 
nicht dem Risiko weiterer Repressalien seitens des Regimes aussetzen wollte, so dass man sich dafür entschied, eine gemäßigtere Fassung zu erstellen. ${ }^{76}$ Der in der Sendung zitierte Teil bezog sich auf die Achtung der ,allgemeinmenschlichen, gottverliehenen Rechte des Menschen“, ohne die die gesamte abendländische Kultur zusammenbrechen würde; gemeint waren hier das natürliche Recht auf Freiheit innerhalb der von Gott und der „Pflicht des Gehorsams gegen die gerechten Gebote der rechtmäßigen Obrigkeit“ gesetzten Grenzen, das Recht auf Leben und Lebenserhaltung, das natürliche Recht auf Privateigentum und Schutz der persönlichen Ehre. ${ }^{77}$ Dieser Teil des gemeinsamen Hirtenbriefes hatte die Punkte der päpstlichen Radioansprache von Weihnachten 1942 vorweggenommen, so dass die Übertragung des Hirtenbriefes mit der thematischen Nähe zur Weihnachtsbotschaft Pius' XII., die in den Medien und in der katholischen Bewegung weite Verbreitung fand, erklärt werden kann. ${ }^{78}$

Der Brief hob ferner den Einsatz der Ordensleute bei der Verteidigung des Vaterlandes hervor; im Feld wie in der Heimat hätten sie „heldenmütig“ ihre Pflicht getan. Keinesfalls aber dürfe den Katholiken zugemutet werden, ihre Treue gegen das Vaterland mit einer Beeinträchtigung ihres eigenen Glaubens unter Beweis stellen zu müssen: „Wir bleiben unserem Vaterlande unverbrüchlich treu, gerade weil wir unserem Heiland und unserer Kirche um jeden Preis die Treue halten. “ Abschließend

76 Vgl. im Detail den Bericht Augustin Röschs vom 23. April 1942 in Volk (wie Anm. 13), Dok. 886, S. 914-921, hier S. 917 f. Den ursprünglichen Hirtenbrief, der nur in den Diözesen Bamberg, Speyer und Würzburg sowie in einigen Pfarreien Regensburgs und Passaus vollständig verlesen wurde, vgl. in Volk (wie Anm. 59), Dok. 751/I, S. 700-704. Den überarbeiteten Text und seine Verbreitung vgl. ebd., Dok. 751/II, S. 705-708. In diesem Punkt ist also Miccoli zu korrigieren, der geschrieben hat, auf die fehlende Reaktion der Reichsregierung sei keine öffentliche Stellungnahme seitens des Episkopats gefolgt; vgl. Miccoli (wie Anm. 5), S. 199.

77 Volk (wie Anm. 59), Dok. 751/II, S. $702 \mathrm{f}$.

78 In seiner Weihnachtsrundfunkansprache 1942 hatte Pius XII. gesagt: „Wer will, dass der Stern des Friedens über dem menschlichen Zusammenleben aufgehe und leuchte, ... der trete ein für die Heilighaltung und praktische Verwirklichung folgender grundlegender Persönlichkeitsrechte: das Recht auf Erhaltung und Entwicklung des körperlichen, geistigen und sittlichen Lebens, ganz besonders auf religiöse Erziehung und Bildung; das Recht zur privaten und öffentlichen Gottesverehrung, einschließlich der religiösen Liebestätigkeit; das grundsätzliche Recht auf Eheschließung und auf Erreichung des Ehezweckes; das Recht auf eheliches und häusliches Gemeinschaftsleben; das Recht zu arbeiten als notwendiges Mittel zur Aufrechterhaltung des Familienlebens; das Recht der freien Wahl des Lebensstandes, also auch des Priester- und Ordensstandes; das Recht zu einer Nutzung an den materiellen Gütern, die sich ihrer sozialen Pflichten und Gebundenheiten bewusst bleibt.“ Vgl. Weihnachtsrundfunkbotschaft „Con sempre“, Absatz 31.2, in: Gerechtigkeit schafft Frieden. Reden und Enzykliken des Heiligen Vaters Pius XII., hg. von Wilhelm Jussen SJ, Hamburg 1946, S. 201-216. Über die Begrenztheit der Erklärung zu den Persönlichkeitsrechten in der Rundfunkansprache Pius' XII. vgl. Daniele Menozzi, Chiesa e diritti umani. Legge naturale e modernità politica dalla Rivoluzione francese ai nostri giorni, Bologna 2012, S. 138 f.; eine andere Lesart, die darin einen Wendepunkt in der Geschichte der Menschenrechte sieht, vgl. in: Samuel Moyn, Christian Human Rights, Philadelphia 2015. 
riefen die Bischöfe den Segen für die Kirche und das Vaterland und einen „ehrenvollen, glücklichen, dauernden Frieden“ an. ${ }^{79}$

Am Tag des Heiligen Petrus Canisius (27. April), der als zweiter Apostel Deutschlands gilt, zog der deutsche Sprecher eine Parallele zwischen der Verfolgung der Kirche im 16. Jahrhundert, gegen die der Heilige angetreten war, und der aktuellen Lage:

„Brothers and sisters! We repeat, we, too, live in a grave hour. The Church is subjected to oppression and persecution. Christians are hard pressed in every way. Divine Law, natural law and human rights given by God are being scorned and neglected.... We must not abandon God in this decisive struggle. “80

Bereits Ende März war das deutsche Volk in einer Sendung über die Pflichten der Katholiken daran erinnert worden, es nehme eine besondere Stellung in der abendländischen christlichen Kultur ein, und wurde ermahnt, seine Wurzeln nicht zu verleugnen. In diesem Zusammenhang zitierte der Sprecher aus der Enzyklika „Mit brennender Sorge“, worin Pius XI. die deutschen Gläubigen aufgefordert hatte, wachsam zu sein, weil die Machthaber den Begriff der christlichen Religion manipuliert hätten. ${ }^{81}$ Nicht nur hier wurde auf die Enzyklika Pius’ XI., die 1937 auf große Resonanz gestoßen war, ausdrücklich Bezug genommen. In einer mehrsprachigen Sendung vom Mai 1943 setzte sich die vatikanische Rundfunkanstalt mit einer Übertragung von Radio Paris auseinander, in der dem Heiligen Stuhl vorgeworfen worden war, er habe Deutschland immer wieder angegriffen, weshalb Göring sich gezwungen gesehen habe, die politischen Aktivitäten des Klerus zu unterbinden. Der deutsche Ansager, wie nach ihm auch sein englischer, polnischer und niederländischer Kollege, hielt dem entgegen, dass die Ablehnung der nationalsozialistischen Philosophie durch die Kirche bereits seit der Enzyklika „Mit brennender Sorge“ bekannt gewesen sei. Und er fügte hinzu, die ganze Welt wisse, wie es um das Schicksal der katholischen Kirche in Deutschland bestellt sei, so dass die aus propagandistischen Motiven verbreiteten Unterstellungen von Radio Paris keiner weiteren Widerlegung bedürften. ${ }^{82}$

79 Aus den abgehörten Mitschriften der BBC geht hervor, dass Radio Vaticana die Langfassung des Hirtenbriefes verlesen hat. Allerdings lässt sich nicht feststellen, wer den ursprünglichen Text übermittelt hat.

80 BBC Written Archives, Daily Digest of Foreign Broadcasts. Vatican City, in deutscher Sprache für Deutschland, 27. April 1943, 21.45 Uhr.

81 Ebd., 29. März 1943, 21.00 Uhr.

82 Ebd., 26. Mai 1943, 21.45 Uhr. Die Sendung wurde zunächst auf Englisch ausgestrahlt, am 28. Mai auf Deutsch, am 1. Juni auf Polnisch und am 3. Juni auf Holländisch. Vgl. auch News, Notes and Texts, in: The Tablet, 5.6.1943, S. 272. Radio Paris stellte während der vierjährigen Besetzung Frankreichs das wichtigste deutsche Propagandainstrument dar. Die im Juli 1940 eingerichtete Propaganda-Abteilung, deren Zentrale sich im Pariser Hotel Majestic befand, bediente sich unter der Bezeichnung Radio Paris dreier staatlicher und zweier privater Rundfunksender. Vgl. Claude Lévy, L'organisation 
Die Programmauswahl, die der Verantwortliche für die deutschsprachigen Sendungen vorgenommen hatte, entsprach dem päpstlichen Wunsch, in dieser Konfliktphase vor allem die nationalen Episkopate sprechen zu lassen. Der an Radio Vaticana ergangene Auftrag bestand offensichtlich darin, ihren Stimmen die größtmögliche Verbreitung auch über die nationalen Grenzen hinaus zu sichern, wobei allerdings solche Themen und Texte behandelt werden sollten, die mit der internationalen Politik des Vatikans zusammenstimmten und nicht nur lokalen Erfordernissen gehorchten.

\section{Schlussfolgerungen}

Mussolinis Sturz, die Unterzeichnung des Waffenstillstandsvertrags und die Besetzung Roms durch die Deutschen verstärkten die wachsame Vorsicht der Kirchenführung, die den ihr nächststehenden Organen („Osservatore Romano“ und Radio Vaticana) die größtmögliche Zurückhaltung auferlegte. Bekanntlich strömte die Bevölkerung bei der Befreiung Roms massenhaft auf den Petersplatz; diese symbolische Geste würdigte Radio Vaticana dadurch, dass die Dankansprache des Papstes auf Deutsch ausgestrahlt wurde. ${ }^{83}$ Das mit Radio Vaticana verbundene Informationsbüro fuhr allerdings in seinen Aktivitäten fort und verbreitete Nachrichten über die Kriegsgefangenen und Vermissten. ${ }^{84}$

Auf die eingangs gestellten Fragen kann nun dahingehend geantwortet werden, dass der Heilige Stuhl sich im Laufe der Jahre mit dem Medium Radio, das im Krieg in gewisser Hinsicht seine Feuerprobe erfuhr, immer vertrauter machte. Pius XII. wurde sich nach und nach seiner Bedeutung bewusst und lernte, wie es sich auf propagandistischer und diplomatischer Ebene einsetzen ließ. Ein strategisches Programm gab es dabei nicht, vielmehr hing es von den jeweiligen Umständen ab, ob der Sender Nachrichten verbreitete oder sich ausschwieg. Mit einer gewissen Sicherheit mag immerhin gesagt werden, dass Radio Vaticana eines der Instrumente darstellte, derer

de la propagande, in: Revue d'histoire de la Deuxième Guerre mondiale 64 (1966), S. 7-28, hier S. 13; Fabrice D'Almeida/Christian Delporte, Histoire des médias en France de la Grande Guerre à nos jours, Paris 2003, S. 80.

83 Am 5. Juni versammelte sich eine zahllose Menge auf dem Petersplatz, um dem Papst zu huldigen und ihm zu danken. Bereits vorher hatte sich die Bevölkerung um ihn geschart, als er nach den Bombenangriffen in die betroffenen Stadtviertel gefahren war. Die Forschung hat zu Recht auf die Bedeutung dieses Phänomens verwiesen. Zum Verständnis dieser Geschehnisse von 1944 trägt eindrücklich Federico Chabod bei, wenn er die Situation mit der Plünderung durch die Westgoten im Jahr 410 und der Kraft der römischen Kirche, sich an Stelle des Kaisertums zum Verteidiger der Bevölkerung gegen die Barbaren aufzuwerfen, vergleicht; vgl. Federico Chabod, L'Italia contemporanea (1918-1948), Torino ${ }^{9} 1961$, S. 125.

84 Inter arma caritas. L'Ufficio Informazioni Vaticano per i prigionieri di guerra istituito da Pio XII (1939-1947), 2 Bde., hg. von Francesca Di Giovanni/Giuseppina Roselli, Città del Vaticano 2004. 
sich der Heilige Stuhl während des Krieges zur Umsetzung seiner diplomatischen Initiativen bediente. Wiederholt überließ es Pius XII. der vatikanischen Sendeanstalt, über untragbare Zustände, Gewaltakte und Übergriffe zu berichten. Der Papst selbst unternahm einige Versuche (wie im Januar 1940), zuweilen - wenn der diplomatische Druck zu groß wurde - machte er einen Rückzieher. Trotz aller Schwierigkeiten und Zensurmaßnahmen, die er selbst zu einem bestimmten Zeitpunkt ergriff, verzichtete er niemals auf die Stimme der Rundfunkanstalt, sei es auch nur, um Gespräche rein religiösen Inhalts oder die Predigten und Hirtenbriefe der Bischöfe auszustrahlen. Die Entscheidung, den Orientierungen der Kirchenoberen weltweite Resonanz zu verleihen, bot nicht nur den deutschen Katholiken die Möglichkeit, die Predigten ihrer Seelsorger kennenzulernen, sondern füllte in gewisser Weise die Lücke, die sich aus der selbstauferlegten öffentlichen Zurückhaltung des Papstes ergeben hatte. Obgleich Pius XII. von den Schwierigkeiten, die Sendungen in den Reichsgebieten zu empfangen, und von den Repressalien gegenüber dem Klerus und den Gläubigen wusste, ordnete er nicht an, die deutschsprachigen Sendungen auszusetzen (und ebensowenig die Übertragungen in den anderen Sprachen, die hier nicht erörtert worden sind). Die neutrale Position, das prekäre Gleichgewicht, das Pius XII. zwischen den beiden Lagern wahren wollte, prägte auch die Radioanstalt, die einerseits vermied, ein offen antinationalsozialistisches Programm zu verfolgen, andererseits aber die Sendungen nicht vollständig einstellte und damit zeigte, dass sie den Forderungen der Achsenmächte nicht nachzugeben gedachte. 